\title{
Roughness induced BL transition measurements in hypersonic flow using infrared thermography
}

\author{
by F. Schrijer*, F. Scarano* and B. van Oudheusden*
}

${ }^{\star}$ Delft University of Technology, Delft, The Netherlands

\begin{abstract}
Roughness induced boundary layer transition is experimentally investigated in the hypersonic flow regime at $M=9$. The primary interest is the possible effect of stepwise geometry imperfections (but also other roughness configurations were studied). Quantitative Infra-Red Thermography (QIRT) is used to measure the surface convective heat transfer and to detect boundary layer laminar-to-turbulent transition. The investigation shows that for a given height of the roughness element, the boundary layer is least sensitive to a step-like disturbance, whereas distributed $3 \mathrm{D}$ roughness was found to be effective in triggering transition. The experimental results have been compared to existing hypersonic transition correlations (PANT).
\end{abstract}

\section{Introduction}

In the design of re-entry vehicles the thermal protection system (TPS) plays a crucial role. During re-entry, the surface temperature of the vehicle increases dramatically as it is subjected to an extremely high convective heat flux. Since the TPS contributes considerably to the weight of a re-entry vehicle and the materials used are relatively expensive, the thermal loads must be carefully predicted in order to minimize the application and dimension of the TPS.

The thermal loads are dominated by the convective heat transfer, which depends on several parameters, namely the re-entry trajectory, vehicle configuration and heat transfer mechanism. Since a turbulent boundary layer acts on the vehicle surface with convective thermal loads up to 5 times higher than for laminar flow [1], the phenomenon of boundary layer laminar to turbulent transition in hypersonic flows is therefore crucial for vehicle design and optimization.

The specific output of transition induced by surface roughness becomes relevant in case that large differences in vehicle surface temperature and the use of different TPS materials introduce significantly different thermal expansion in turn causing the vehicle surface to deform or expose discontinuities such as gaps and steps. Extensive investigation performed in the 60's (PANT) [2], [4] led to the formulation of experimental databases for the prediction of hypersonic roughness induced boundary layer transition. The resulting prediction criteria are based on the correlations fitting the data. The scarce availability in this type of data and the difficulty to tackle the problem from a computational point of view makes the mentioned correlation the primary source of reference and therefore it is applied in many cases.

The present study is motivated by the specific type of roughness to be investigated, namely a backward and forward facing step. Two objectives are presented: the first is to verify in which conditions a step-like disturbance triggers boundary layer transition; the second is to verify the validity of the existing correlations for this type of surface disturbance on a simplified (generic) blunt-nosed object in hypersonic flow. 


\section{Experimental setup and data reduction}

The experiments are performed in the Hypersonic Test Facility Delft (HTFD) that operates on the Ludwieg tube principle [3]. The tunnel is equipped with a conical nozzle, producing a free stream Mach number $M_{\infty}=9$. The unit Reynolds number can be varied from $4 \times 10^{6}$ to $14 \times 10^{6} \mathrm{~m}^{-1}$ when the total pressure of the wind tunnel is varied between 50 and 100 bar. The tunnel is operated at a total temperature of $T_{t}=770 \mathrm{~K}$ to avoid air condensation in the free stream. The typical run time of the tunnel is $100 \mathrm{~ms}$.

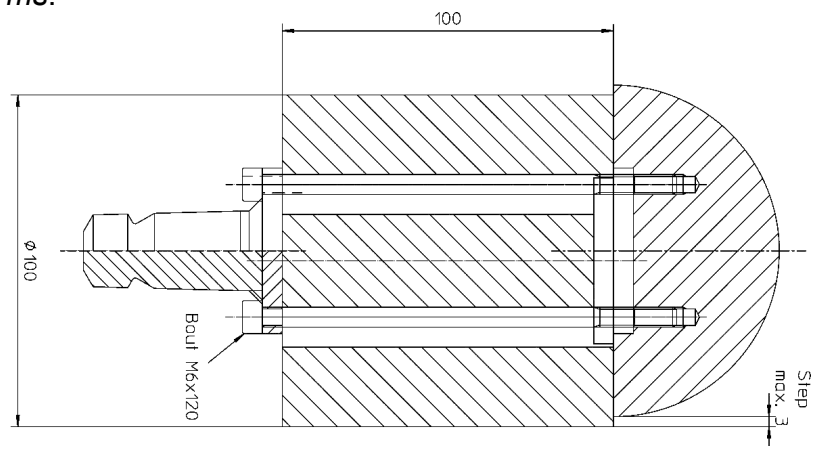

Fig. 1. Model drawing (dimensions in $\mathrm{mm}$ )

The wind tunnel model used in the experiments consists of a hemispherical nose and a cylindrical after-body; the model geometry and dimensions are given in figure 1. The model is built in Makrolon, a polycarbonate material with favourable thermal properties for the application of the quantitative infrared thermography technique. Its low thermal conductivity $(k=0.15 \mathrm{~W} / \mathrm{K} \mathrm{m})$ and the relatively high surface emissivity coefficient $(\varepsilon=0.8)$, make the material a successful choice for the current application [5]. Moreover, Makrolon can withstand temperatures as high as $120^{\circ} \mathrm{C}$ with unaltered properties, which is below the maximum expected temperature during the thermal transient.

The roughness elements used in the experiments are a backward facing and forward facing step obtained with a relative shift of the nose with respect to the body axis, as well as separately attached elements like tripping wires, sandpaper and patches of small spheres (grit). Step heights of $h=[-3.2,-1.0,1.0,3.2] \mathrm{mm}$ were studied, where '-' denotes a backward facing and ' + ' denotes a forward facing step. The sandpaper grain size was varied between $k=0.26 \mathrm{~mm}$ and $k=0.98 \mathrm{~mm}$. To achieve a distributed roughness with a higher roughness height, spherical steel shot (grit) was used with a diameter of $k=1.5 \mathrm{~mm}$ and $k=2.0 \mathrm{~mm}$. The length of the patches in flow direction was respectively $20 \mathrm{~mm}$ (sandpaper) and $15 \mathrm{~mm}$ (grit). The tripping wires were glued to the model nose at different angles from the stagnation point $\phi=\left[30^{\circ}, 45^{\circ}, 60^{\circ}, 90^{\circ}\right]$, the wire diameter is $d=1 \mathrm{~mm}$.

The IR system used to obtain the surface temperature variation consists of an Agema Thermovision 880 LWB infrared scanner with a BRUT data acquisition system. The scanner is operated in line-scan mode where a single horizontal profile of the temperature is recorded repeatedly at a frequency of $2.5 \mathrm{kHz}$ along a meridian line of the model. 
The time evolution of the local temperature $T_{s}(s, t)$ in a one-dimensional semiinfinite solid is related to the local convective heat transfer $q_{s}(s)$ to the surface. When $q_{s}(s)$ is assumed constant in time, the temperature evolution reads as:

$$
T_{s}(s, t)=T_{i}+2 q_{s}(s) \sqrt{\frac{t}{\pi \rho c k}}
$$

where $T_{i}$ is the initial (uniform) temperature of the model and $s$ is the position on the model, measured along its surface. From the above expression it follows that the ratio of the heat transfer relative to that at the stagnation point (where the maximum heat transfer is expected), can be obtained directly by normalizing the instantaneous temperature profiles:

$$
\bar{T}(s, t)=\frac{T_{s}(s, t)-T_{i}}{T_{s, \text { stag }}(t)-T_{i}}=\frac{q_{s}(s)}{q_{s, \text { stag }}} .
$$

The validity of this approach was assessed by verifying that the temperature profiles obtained at different times were self-similar, which indicated that the normalized temperature function $\bar{T}(s, t)$ as defined above, is indeed independent of time.

\section{IR Measurements}

In figure 2 the heat transfer distribution is given for a smooth model and a model having either a backward or forward facing step at the nose-body junction. At the stagnation point the normalized heat transfer has a value of one and decreases moving downstream due to boundary layer growth. In case of the forward facing step $(h=+3.2 \mathrm{~mm})$ a local decrease in heat transfer can be appreciated upstream of the step caused by flow separation. The following peak is due to the exposure of the sharp step edge to the incoming flow. Further downstream the heat transfer approaches the heat transfer of the smooth model.

For the backward facing step only a local decrease in heat transfer can be observed, which is ascribed to flow separation behind the step. From the reattachment point on, the heat transfer profile follows the profile obtained for the smooth model.

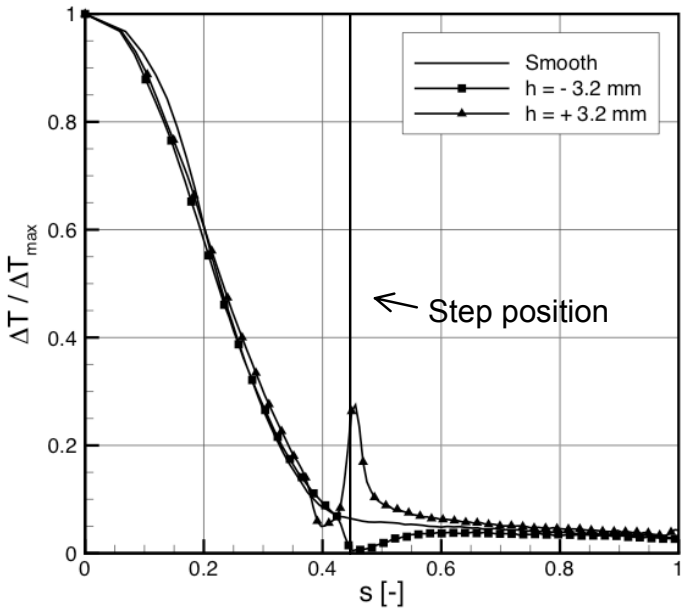

Fig. 2. Heat transfer distribution for a model with a surface step. 
For the case of 3D distributed roughness (sandpaper, grit) the heat transfer measurements are depicted in figure 3 which shows only the evaluation downstream of the element. The measurements show an increase in heat transfer when the roughness height increases above $0.5 \mathrm{~mm}$. When $k<0.5 \mathrm{~mm}$ no difference in heat transfer can be appreciated. For the case of $k=0.74 \mathrm{~mm}$, the heat transfer is twice the laminar value, which is ascribed to turbulent mixing in the boundary layer. Since for the largest roughness heights the heat transfer still increases with roughness height it cannot be concluded that a completely turbulent boundary layer state is reached.

Applying steel shot (grit) on the model surface, an even higher roughness height could be obtained and the heat transfer was found to increase further. At $k=2.0 \mathrm{~mm}$ the heat transfer increases by a factor three with respect to the laminar case.

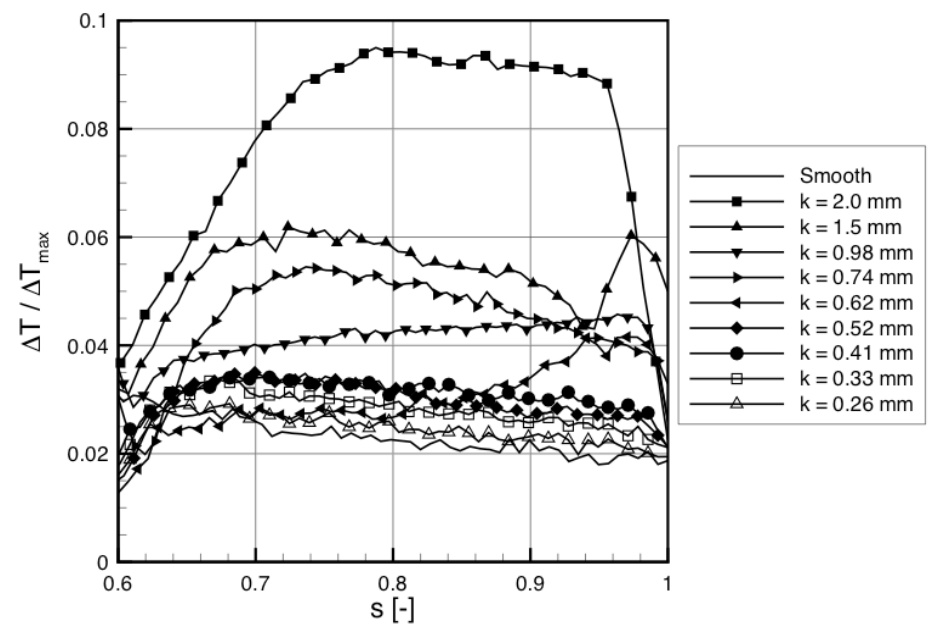

Fig. 3. Heat transfer distributions downstream of the roughness elements for sandpaper and grit roughness.

The heat transfer profiles for the 2D (wire) and 3D (grit-line) roughness lines are given in figure 4 . Adding the wire to the model only causes a small increase in heat transfer with respect to the smooth model case. The increase is identical for all angular positions, except for the wire at $60^{\circ}$ (which seems to give little effect), the reason for this is unclear. When changing from a 2D (wire) to a $3 D$ (grit-line) disturbance, the heat transfer increases significantly: compare the wire at $90^{\circ}$ with the grit line which is at the same position. Apparently, roughness with an (3D) azimuthal varying roughness height is much more effective in perturbing the boundary layer. 


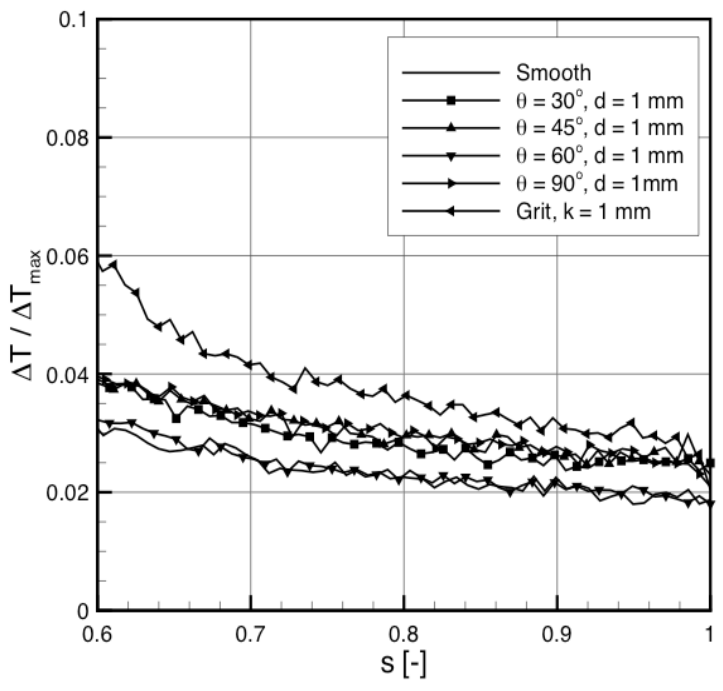

Fig. 4. Heat transfer distribution for the wire and grit-line roughness elements

\section{Results}

Several studies have been devoted to the study of boundary layer transition correlations. In these correlations, a transition parameter is correlated to a flow disturbance parameter. Transition is assumed to occur when, at a given value of the disturbance parameter, the transition parameter exceeds a critical value. The critical value of a transition parameter (typically the Reynolds number based on the boundary layer momentum thickness) is based on the hypothesis that above a critical Reynolds number, vortices shed in the wake of the roughness element(s) trigger the boundary layer instability [4].

A correlation for hypersonic roughness induced transition was developed in the Passive Nose-tip Technology program (PANT). The transition parameter taken is the Reynolds number based on the momentum loss thickness, while the disturbance parameter is a combination of the roughness height to momentum loss thickness ratio and the wall to boundary layer edge temperature ratio:

$$
R e_{\theta, \mathrm{tr}}=a\left(\frac{k}{\theta} \frac{T_{e}}{T_{w}}\right)^{n}
$$

The temperature ratio is included to take into account the (de)stabilising effect of temperature variations across the boundary layer. The coefficients $n$ and a were obtained applying curve fits to experimental data. Several choices have been used notably $a=500$ with $n=-1.5$ and $a=215$ with $n=-0.7$ [2] see figure 5 .

The results from current experiments are depicted in figure 5 . The values for the boundary layer parameters for the different roughness locations are obtained from a CFD calculation considering an isothermal wall $\left(T_{w}=290 \mathrm{~K}\right)$. The computation was performed with LORE, a finite volume second order Navier-Stokes solver [6]. The effective roughness location is taken as the most downstream position of the roughness element. The experimental results are labelled ' $L$ ' laminar ( $\left.q \approx q_{\text {smooth }}\right)$, ' $t$ ' transitional $\left(q_{\text {smooth }}<q<2 q_{\text {smooth }}\right)$ and ' $T$ ' turbulent $\left(q>2 q_{\text {smooth }}\right)$. 


\section{http://dx.doi.org/10.21611/qirt.2004.090}

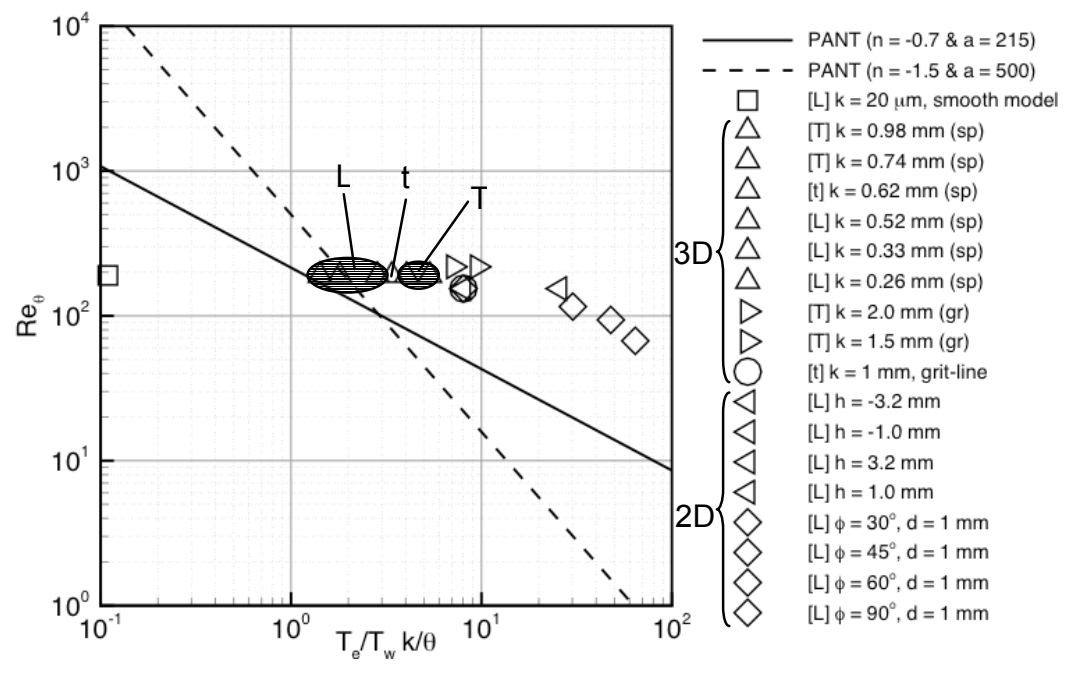

Fig. 5. Current experiments compared with PANT correlation. [L] = laminar, [t] $=$ transitional, $[T]=$ turbulent,$s p=$ sandpaper and $g r=$ grit.

From figure 5 it is clear that the 3D type of roughness introduces a behaviour in good agreement with the PANT correlation. The experiments performed below the critical disturbance parameter returned laminar flow behaviour. Moreover when the critical value (according to the PANT correlation) is reached, the experiments returned transitional and turbulent conditions associated to increasing values of the heat transfer.

The situation for the 2D type of roughness is rather different. Basically no experiment returned evidence of turbulent flow conditions in the investigated range of the disturbance parameter. It is therefore concluded that the $2 \mathrm{D}$ isolated roughness disturbs the incoming laminar boundary layer to a much lesser extent with respect to the equivalent (i.e. with the same roughness height) 3D type of distributed roughness. This is caused by the fact that the two-dimensional roughness elements introduce 2D disturbances. This $2 \mathrm{D}$ disturbance must then first be converted into a 3D fluctuation before transition is triggered. However, the 3D roughness elements directly produce 3D disturbances (stream-wise vortices), which affect flow mixing and trigger boundary layer transition more effectively.

\section{Conclusions}

The influence of surface roughness on boundary layer transition in hypersonic flow has been investigated with infrared thermography on a hemisphere-cylinder configuration, considering both three-dimensional distributed and isolated roughness as well as two-dimensional disturbances. Transition was detected by means of an increase in heat transfer. The infrared thermography implemented in the passive configuration revealed to be a reliable and accurate tool for transition detection and characterisation in the short-duration wind tunnel facility. The measured data confirmed the applicability of existing empirical hypersonic transition correlations for three-dimensional distributed roughness, while the agreement is not found for the case of the two-dimensional step configuration. The two-dimensional roughness elements were found to be less effective in promoting transition with respect to the three-dimensional elements. 


\section{REFERENCES}

[1] Anderson, J.D., Hypersonic and High Temperature Gas Dynamics, AIAA, 2000.

[2] Batt, R.G. and Legner, H.H., A Review of Roughness-Induced Nosetip Transition, AIAA Journal, Vol. 21, No. 1, 1983, pp 7-22.

[3] Friehmelt, H., Koppenwallner, G. and Müller-Eigner, R. Calibration and first results of a redesigned Ludwieg expansion tube, AIAA-paper 93-5001, AIAA, 1993

[4] Reda, D.C., Review and Synthesis of Roughness-Dominated Transition Correlations for Reentry applications, Journal of Spacecraft and Rockets, Vol. 39, No. 2, 2002, pp. 161-167.

[5] Schrijer, F.F.J., Scarano, F and Van Oudheusden, B.W., Experiments on Hypersonic Boundary Layer Separation and Reattachment on a Blunted ConeFlare using Quantitative InfraRed Thermography, AIAA-paper 03-6967, AIAA, 2003.

[6] Walpot, L.M.G.F.M, Development and Application of a Hypersonic Flow Solver, Ph.D. Thesis, Delft University of Technology, 2002. 